EPJ Web of Conferences 43, 05012 (2013)

DOI: $10.1051 /$ epjconf/20134305012

(C) Owned by the authors, published by EDP Sciences, 2013

\title{
Identification of PG $2303+243$ pulsation modes
}

\author{
E. Pakštienè $\dot{a}^{2}$ \\ Vilnius University Institute of Theoretical Physics and Astronomy, A. Goštauto 12, \\ 01108 Vilnius, Lithuania
}

\begin{abstract}
For this study we used total of 40 possibly independent frequencies of pulsation of a cool DA variable star PG 2303+243 observed in 2004 and 2005. Using period spacing diagrams we indentified 10 $l=1$ and $14 l=2$ modes with a mean period spacing of $52.00 \mathrm{~s}$ for $l=1$ modes and $31.85 \mathrm{~s}$ for $l=2$ modes.
\end{abstract}

\section{INTRODUCTION}

PG $2303+243$ is a cool DA variable (also called ZZ Ceti) star having a rich pulsation spectrum and variable amplitudes. Using photometric observations of PG 2303+243 from 2004 we detected 24 possible independent frequencies within a period interval of 206-2234 s [1]. During 5 consecutive nights in 2005 observations of PG2303+243 were repeated at Moletai Obs. (Lithuania) and 16 additional possible independent frequencies were detected. For this study we used a total of 40 frequencies (see Fig. 1). 25 of them were identified as $l=1$ and $l=2$ modes (Table 1).

\section{MODES IDENTIFICATION}

The Fourier transform (FT) spectra of PG 2303+243 obtained in 2004 and 2005 are clearly different (Table 1). Although some peaks can be found at about the same frequency, they have different amplitudes. The most stable mode is detected at $1622 \mu \mathrm{Hz}(616 \mathrm{~s})$. Even though its amplitude from 2004 to 2005 decreased by almost twice, it still was one of the strongest peaks in the FT spectrum.

Pakštiene et al. [1] claimed that the mode at $616 \mathrm{~s}$ is a $l=1$ mode. We compared our results with those obtained by Romero et al. [2]. The latter presented an asteroseismological analysis of 44 bright ZZ Ceti stars based on a new set of fully evolutionary DA white dwarf models characterized by detailed chemical profiles from the centre to the surface [2].

Romero et al. [2] used only four main frequencies of PG 2303+243 with the largest amplitudes to estimate the physical parameters for the star and fixed the harmonic degree to be $l=1$ for two main modes, 616.4 and $965.3 \mathrm{~s}$, while 863.8 and $394.4 \mathrm{~s}$ were allowed to be of degree $l=1$ or 2 .

Our study of period spacings pattern in FT spectrum of PG 2303+243 confirmed $l=1$ for 616.4 and $965.3 \mathrm{~s}$, and $l=2$ for $394.4 \mathrm{~s}$, while the mode at $863.8 \mathrm{~s}$ was identified as a $l=1$ mode. Another closely spaced mode at $873.2 \mathrm{~s}$ most likely is a $l=2$ mode. Using $394.4,616.4$ and $965.3 \mathrm{~s}$ as datum-periods we estimated $l$ values for most of the modes (see Fig. 1 and Table 1). We were not sure about two modes at 778.5 and $784.5 \mathrm{~s}$. Both modes had high amplitudes, but only one of them could fit our period spacing pattern for $l=2$. As was written before, these two modes may belong to a multiplet with a central peak at about $778.5 \mathrm{~s}$. We labelled this mode a $l=2$ mode. If the central peak of the multiplet were at $784.5 \mathrm{~s}$,

\footnotetext{
a e-mail: erika.Pakštienè@tfai.vu.lt
}

This is an Open Access article distributed under the terms of the Creative Commons Attribution License 2.0, which permits unrestricted use, distribution, and reproduction in any medium, provided the original work is properly cited. 


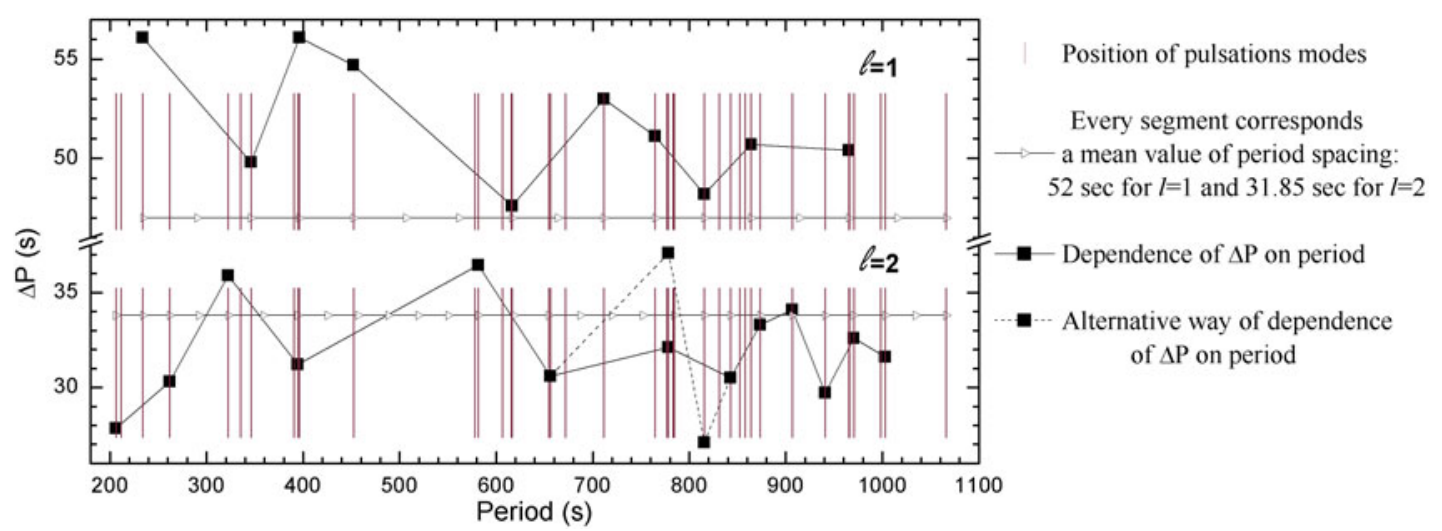

Figure 1. Diagram of "forward" period spacing for PG $2303+243$.

Table 1. List of 25 identified modes of PG $2303+243$.

\begin{tabular}{llllllllllll}
\hline$P(\mathrm{~s})$ & $A(\mathrm{mma})$ & $\Delta P(\mathrm{~s})$ & $l$ & $k$ & year & $P(\mathrm{~s})$ & $A(\mathrm{mma})$ & $\Delta P(\mathrm{~s})$ & $l$ & $k$ & year \\
\hline 206.1 & 2.3 & 27.8 & 2 & 3 & 2004 & 778.5 & 20.9 & 32.1 & 2 & 21 & 2005 \\
234.1 & 3.2 & 56.1 & 1 & 1 & 2004 & 784.5 & 14.5 & - & $(2)$ & $(21)$ & 2005 \\
261.8 & 2.3 & 30.3 & 2 & 5 & 2004 & 815.6 & 12.4 & 48.2 & $1 ;(2)$ & $12 ;(22)$ & 2005 \\
322.5 & 3.1 & 35.9 & 2 & 7 & 2004 & 842.7 & 5.3 & 30.5 & 2 & 23 & 2005 \\
346.3 & 3.8 & 49.8 & 1 & 3 & 2005 & 863.8 & 7.4 & 50.7 & 1 & 13 & 2004 \\
394.4 & 7.3 & 31.2 & 2 & 9 & 2004 & 873.2 & 3.9 & 33.3 & 2 & 24 & 2004 \\
396.1 & 3.7 & 56.1 & 1 & 4 & 2005 & 906.5 & 8.3 & 34.1 & 2 & 25 & 2005 \\
452.2 & 2.2 & 54.7 & 1 & 5 & 2004 & 940.6 & 7.5 & 29.7 & 2 & 26 & 2005 \\
581.7 & 4.4 & 36.4 & 2 & 15 & 2005 & 965.3 & 19.7 & 50.4 & 1 & 15 & 2004 \\
616.4 & 31.3 & 47.6 & 1 & 8 & 2004 & 970.3 & 5.1 & 32.6 & 2 & 27 & 2005 \\
656.0 & 2.7 & 30.6 & 2 & 17 & 2004 & 1002.9 & 5.4 & 31.6 & 2 & 28 & 2005 \\
711.5 & 2.0 & 53.0 & 1 & 10 & 2004 & 1066.1 & 2.2 & - & $1 ; 2$ & $17 ; 30$ & 2004 \\
764.5 & 6.2 & 51.1 & 1 & 11 & 2005 & & & & & & \\
\hline
\end{tabular}

it would fit the period spacing pattern of $l=2$ with no big difference. The mode at $1066.1 \mathrm{~s}$ may be both a $l=1$ or a $l=2$. The mode at $815.8 \mathrm{~s}$ is most likely a $l=1$ mode, but it may be a $l=2$ as well, as it is shown in Fig. 1 using dashed line.

The $k$ values for $l=1$ modes fit very well the values given by Romero et al. [2] with the $k=1$ mode at $234.1 \mathrm{~s}$, while $\mathrm{k}$ values for $l=2$ may be slightly different. According to Romero et al. [2], the $k$ value for the mode at $206.1 \mathrm{~s}$ was set up as equal to 3 . As this mode is the first in the list of periods, it may be a $k=1$ as well (we need to check this with additional analysis).

We couldn't recognise modes of period longer than $1066.1 \mathrm{~s}$ because the spaces between them are too big to find unambiguous $l$ values.

\section{CONCLUSIONS}

As a result of this study we analysed the PG $2303+243$ pulsation spectrum and found $10 l=1$ and $14 l=2$ modes with a mean period spacing of $52.00 \mathrm{~s}$ for $l=1$ modes and $31.85 \mathrm{~s}$ for $l=2$ modes. As Romero et al. [2] used only four main frequencies of PG $2303+243$ with the largest amplitudes to 
Ageing Low Mass Stars: From Red Giants to White Dwarfs

estimate the physical parameters of the star, we suggest to calculate a model of PG $2303+243$ using more pulsation modes with known $l$ and $k$ values, determined in this study using observations obtained in 2004 and 2005.

\section{References}

[1] E. Pakštienè, J.-E. Solheim, G. Handler et al., MNRAS 415, (2011) 1322

[2] A.D. Romero, A.H. Corsico, L.G. Althaus et al., MNRAS 420, (2012) 1462 